\title{
Review of the EP Activities of US Academia
}

\author{
Compiled and Edited by:
}

Lyon B. King

Michigan Technological University, Houghton, MI 49931-1295

\begin{abstract}
This paper presents a broad overview of electric space propulsion research being performed at U.S. universities during 2004. University research serves a critical role investigating fundamental issues associated with the science and engineering of space propulsion systems, while serving to educate and train the workforce of the future. Academic research is vibrant, with eleven research groups from nine universities contributing to this paper reporting on devices with power levels ranging from milliwatts to megawatts. Summaries are presented from Colorado State University, Massachusetts Institute of Technology, Michigan Technological University, Princeton University, Texas Tech, University of Illinois at Urbana/Champaign, University of Michigan, Virginia Polytechnic Institute, and Worcester Polytechnic University.
\end{abstract}

\section{Colorado State University \\ Azer P. Yalin, John D. Williams, and Paul J. Wilbur \\ Department of Mechanical Engineering \\ Fort Collins, CO 80525 \\ pwilbur@vines.colostate.edu}

The research at Colorado State University is headed by Profs. Azer Yalin, John Williams, and Paul Wilbur. Research concentrates on fundamental problems related to ion thrusters, with a particular focus on studying sputter erosion processes and ion optics assemblies. Sputter erosion studies are performed with both cavity ring-down spectroscopy (CRDS) and a quartz crystal microbalance (QCM) system. Ion optics studies use sub-scale grids (gridlets) that contain a fraction of the total number of holes associated with a full ion optics system. In this submission we detail the recent CRDS work.

A novel laser-based system for rapid and ultra-sensitive sputter erosion measurements by cavity ring-down spectroscopy (CRDS) is being developed. The technique may prove to be very suitable for accelerated life test (erosion rate) measurements, which are becoming increasingly important as mission durations increase. The detection system uses a Nd:YAG pumped Optical Parametric Oscillator (OPO) laser to study sputtered particles in a test facility comprised of an ion beam (a modified structurally integrated thruster [1] obtained from NASA) and target within a vacuum chamber. A schematic diagram of the system is shown in Figure 1. We have recently demonstrated sensitive measurements of sputtered molybdenum and titanium [2]. Figure 2 shows a measured spectrum of titanium sputtered by an $18 \mathrm{~mA}$ argon ion beam. The measured lines are in the vicinity of $395 \mathrm{~nm}$ and are due to transitions from three different lower states (fine structure splitting of the ground state) to three different upper states. The measured titanium number densities exhibit the expected linear behavior versus beam current, and are in reasonable agreement with values found from a simple sputtering model. Our current configuration yields line density detection limits of $\sim 7 \times 10^{7} \mathrm{~cm}^{-2}$ and $\sim 6 \times 10^{6} \mathrm{~cm}^{-2}$ for titanium and molybdenum respectively; levels which are significantly lower than those required for characterizing electric propulsion devices during life tests.

We are working to demonstrate the use of CRDS to measure particle velocity, which in combination with number density can yield flux and erosion rate. (Velocity fields may also be found from sputter modeling or other measurement techniques such as laser induced fluorescence). Our CRDS velocity measurement approach is based on Doppler effects (shifts and broadenings) in the measured spectral lineshape. Another area of activity is to extend the CRDS approach to other materials, especially carbon. Recent research has shown that for low energy ions, atomic $\mathrm{C}$ comprises only a small fraction of the total sputtered carbon [3]. While $\mathrm{C}$ is difficult to measure with CRDS (since its transitions are too far in the UV), it is possible to measure species such as $\mathrm{C}_{2}, \mathrm{C}_{3}$, etc. For example, $\mathrm{C}_{2}$ can be measured via its $\operatorname{Swan}(0,0)$ band in the vicinity of $515 \mathrm{~nm}$. A set of CRDS experiments to study carbon sputtering is being planned. 


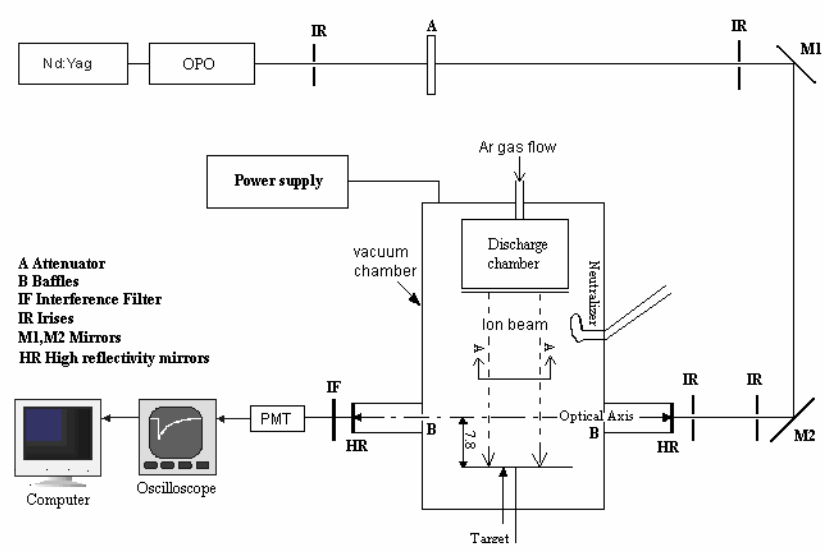

Figure 1. Schematic diagram of CRDS detection system and sputter test facility.

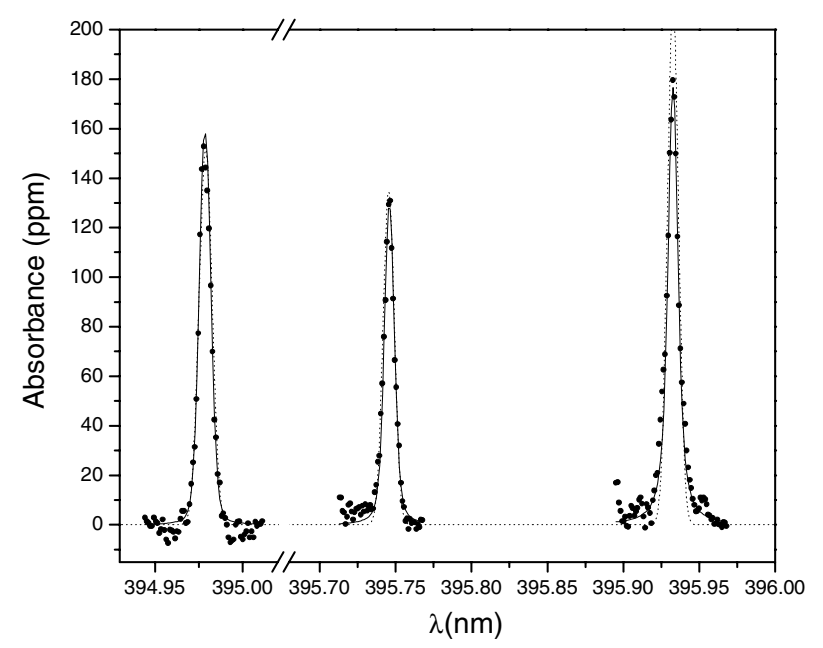

Figure 2. Titanium absorbance spectrum recorded by CRDS. Symbols are experimental data, solid line shows Voigt profiles fitted to the peaks, and dotted line shows a simulated spectrum.

\section{References}

1. W.R. Hudson and B.A. Banks, AIAA Paper 731131, 10th AIAA Electric Propulsion Conference, Lake Tahoe, NV (1973)

2. V. Surla, P.J. Wilbur, J.D. Williams, M. Johnson, A.P. Yalin, "Sputter Erosion Measurements of Titanium and Molybdenum by Cavity Ring-Down Spectroscopy”, Review of Scientific Instruments, in press

3. Doerner, R.P., D.G. Whyte, and D.M. Goebel, Jnl. App. Phys. 93(9): p. 5816-5823 (2003)

\section{Massachusetts Institute of Technology \\ Manuel Martinez-Sanchez \\ Department of Aeronautics and Astronautics \\ Cambridge, MA 02139 \\ mmart@mit.edu}

During the past year, our group has been active in several electric propulsion areas. The main achievements are briefly summarized below.

Electrospray (colloid) propulsion: Pure ion emission from externally wetted solid needles (Figure 3b) [1] (high propulsive efficiencies $>90 \%$, low divergence angles $<20^{\circ}$ ) has been demonstrated, together with the elimination of electrochemical decomposition/corrosion using voltage alternation at low frequencies [2] (square wave $\mathrm{AC} \sim 1 \mathrm{~Hz}$ ) and the production of bipolar ion beams to achieve charge neutrality without using external cathode emitters [3]. The first generation of microfabricated electrospray laboratory thrusters has been preliminary tested: internally fed linear 1D and 2D planar arrays (Figure 3a) [4]. Moderate voltage electrospray emission from non-wettable dielectric surfaces [5] has also been demonstrated (Figure 3c). Our numerical cone-jet code is now able to reproduce observations regarding current versus flow and voltage and minimum stable flow conditions for a variety of liquids and flow rates [6].
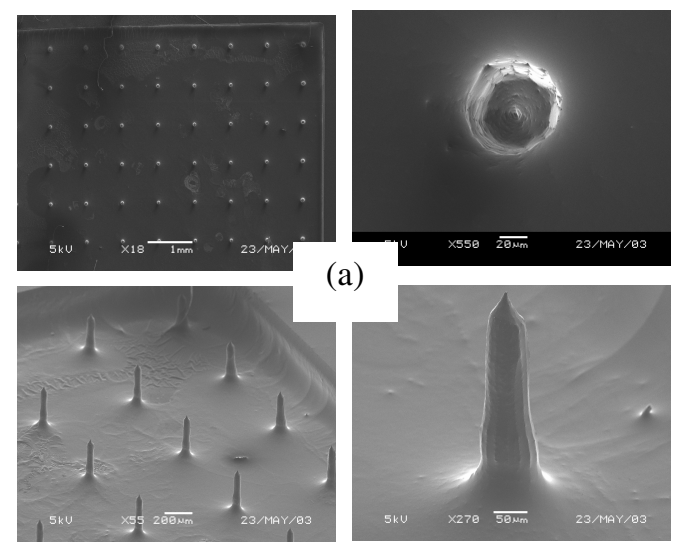

(a)
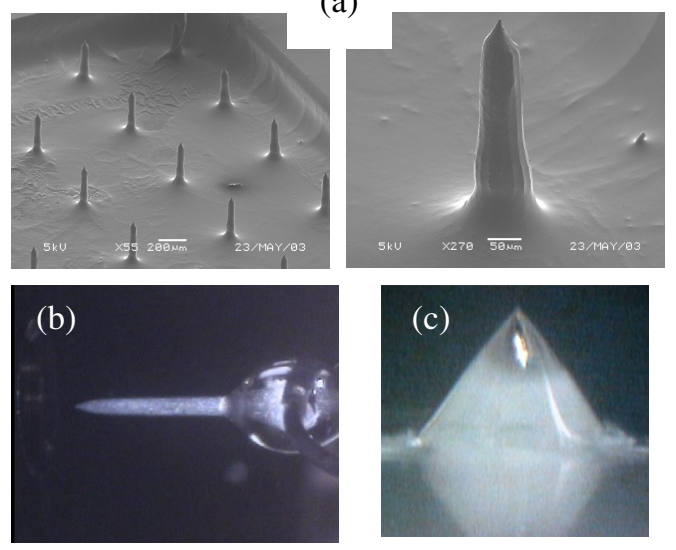

Figure 3. (a) 4 views of a microfabricated, externally wetted needle array, (b) a single tungsten ionic liquid ion source (ILIS) working without suffering electrochemical decomposition and (c) a Taylor cone formed at the exit of a small hole drilled over an otherwise featureless Teflon surface. 
Modeling of Hall Effect Thrusters: PIC modeling of the operation of the P5 SPT at 3 and $5 \mathrm{~kW}$ has yielded correct peak ionization and densities near anode and revealed the important role of magnetic mirroring in P5 , responsible for forcing simulated plasma to outer wall and for lower sheath strength compared to the classical value (Figure 4a). A separate study of the electron transport indicate that the simulated mobility agrees with mobility calculated from collision frequencies.

AQUILA Plume model: Numerical simulations are run to verify if the wake structure behind a shield could be reproduced. AQUILA is well-suited to handle the wake region where quasineutrality breaks down. In order to model the experimental conditions as faithfully as possible, a new source model representing the PPPL-90, the thruster used in the experiment, is created using results from HPHall.

PIC Code parallelization: The objective is to obtain more accurate and extensive results of the MIT Full-PIC simulation by using larger numbers of particles, more resolved grids, a more physical free-space permittivity constant, and a more realistic mass ratio while decreasing computational time, which has dropped from 97 to 27 hrs using 1 and 8 processors respectively.

Bare electrodynamic tether simulation: A faster kinetic model $[7,8]$ based on FFT solvers is used to obtain the potential distribution with correction for the internal (tether) boundary conditions; multiple tether and multiple ions with real mass capabilities (Figure $4 b$ ); large simulation domain $(\sim 10 \mathrm{~m}$ size $)$ for resolution of the wake field region and a multi-scale algorithm to allow wide range of tether voltages.

VASIMR modeling: Abrupt transition to a gas burn regime is confirmed [9] by our 0-D power and mass balance model that takes into account essential plasma chemistry, radiation and wall losses, axial transport and ambipolar acceleration effects (Figure 4c). The development of a self-consistent model of the helicon discharge embracing major physical issues such as RF wave excitation and absorption by plasma, gas ionization and transport, wall and volumetric collisions of multiple plasma and gas species is underway.

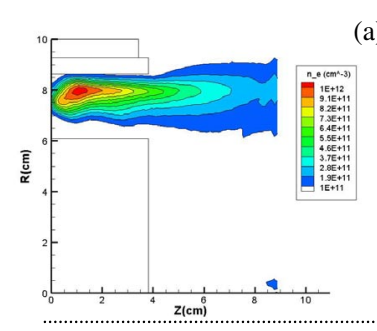

(a) (b)

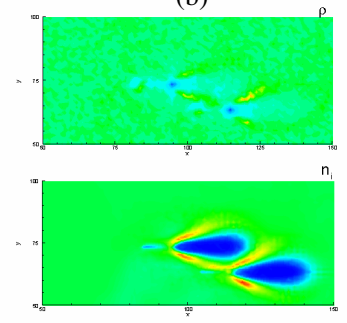

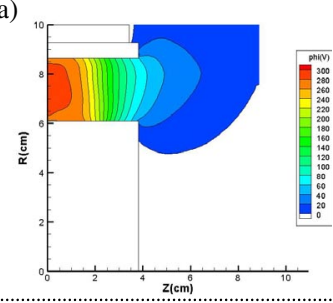

(c)

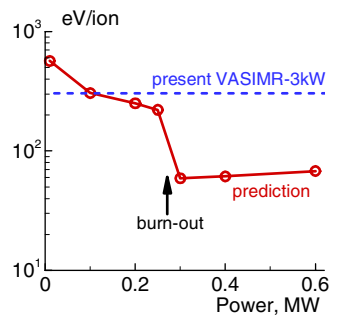

Figure 4. (a) electron density (left) and potential in P-5 SPT, (b) two bare ED tethers and (c) prediction of propellant burnout and transition to low ionization cost regime for VASIMR.

\section{References}

1. P. Lozano and M. Martinez-Sanchez, Ionic Liquid Ion Sources: Characterization of Externally Wetted Emitters, submitted to the Journal of Colloid and Interface Science JCIS-04-498

2. P. Lozano and M. Martinez-Sanchez, Ionic Liquid Ion Sources: Suppression of electrochemical reactions using voltage alternation, subm. to the Journal of Colloid and Interface Science JCIS-04348R1

3. P. Lozano and M. Martinez-Sanchez, Externally Wetted Ionic Liquid Thruster, 4th International Spacecraft Propulsion Conference, Chia Laguna, Sardinia, Italy (2004).

4. L. Velazquez, PhD Thesis MIT 2004.

5. P. Lozano and M. Martinez-Sanchez, Electrospray emission from nonwetting flat dielectric surfaces, in Press at the Journal of Colloid and Interface Science, 2004.

6. J. Carretero, M. Martinez-Sanchez and F. Higuera, Numerical Simulation of a single-emitter Taylor cone electrospray, submitted to the J. of Fl. Mech.

7. O.Batishchev and M.Martinez-Sanchez, Proc. 18th ICNSP, p.379, Cape Cod, USA, September 7-11, 2003

8. J.-M.Deux, O.Batishchev, M.Martinez-Sanchez, Advanced Kinetic Model for Electrodynamic Space Tether, p.162, 4th ISPC, Italy, 2-9 June 2004.

9. O. Batishchev, K. Molvig, F. Chang-Diaz, J. Squire, Study of Gas Burn-out Regime in the VASIMR Helicon Plasma Source, paper O-3.3D, 4p, 30th EPS CFPP Conference, St. Petersburg, Russia, July 7-11, 2003; O. Batishchev, K. Molvig, p.191, 4th ISPC, , Italy, 2-9 June 2004. 


\section{Michigan Technological University}

\author{
Lyon B. King \\ Ion Space Propulsion Laboratory \\ Department of Mechanical Engineering \\ Houghton, MI 49931 \\ lbking@mtu.edu
}

The Isp Lab at MTU was established in 2001 and is currently working on a variety of projects relating to electric propulsion. Research is supported by AFOSR, NASA-GRC, NSF, Aerojet, and the Michigan Space Grant Consortium.

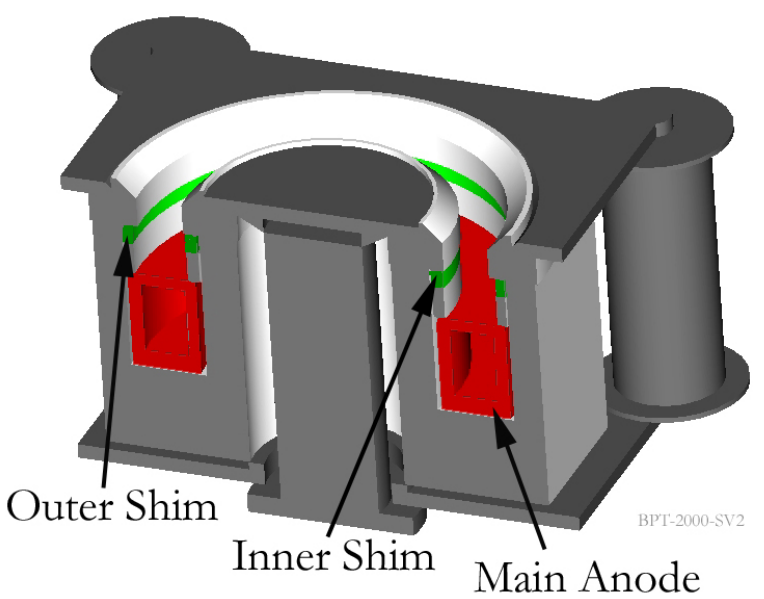

Figure 5. Segmented anode Hall thruster for direct evaporation of bismuth into discharge chamber. The Main Anode serves as a liquid bismuth reservoir and vapor diffuser. The shims serve as current dumps that have the capability to control Main Anode temperature and, hence, evaporation rate.

Bismuth Hall Thruster. Bismuth has both physical and economic advantages over xenon when used as a propellant. Its atomic mass is higher (209 amu vs. 131 amu), its ionization energy is lower ( $7 \mathrm{eV}$ vs. $12 \mathrm{eV})$, it is orders of magnitude cheaper ( $\$ 8 / \mathrm{kg}$ vs. $\$ 7,700 / \mathrm{kg}$ ) and it doesn't require cryogenic pumps for ground testing. MTU is developing a novel bismuth mass-flow control system wherein liquid bismuth is directly evaporated from the anode and no external heaters are used. The evaporation rate is controlled via powersharing segmented anodes that divide the discharge current between the evaporative main anode and inert shims. The thermal control concept has been proven using xenon propellant and efforts are underway to demonstrate with bismuth in 2005. An image of the segmented-anode thruster is shown in Figure 5. A dedicated bismuth testing facility has been constructed. The centerpiece is a 2-m-diameter by 4-m-long tank evacuated through three 2,000-liter-per-second turbopumps.
Nanotube Erosion Studies. Preliminary experiments have been performed to assess the sputter-erosion rate of vertically aligned carbon nanotubes. Early results indicate that nanotube films may have erosion resistance comparable with CVD diamond.

Microscale Quadrupole Mass Spectrometer. The Isp lab has developed a quadrupole mass spectrometer with characteristic dimension of $\sim 10^{-6} \mathrm{~m}$. The device is fabricated from $\mathrm{GaAs} / \mathrm{AlGaAs}$ using rectangular cantilevered electrodes in place of cylindrical rods. Prototype devices have been fabricated and are shown below in Figure 6.

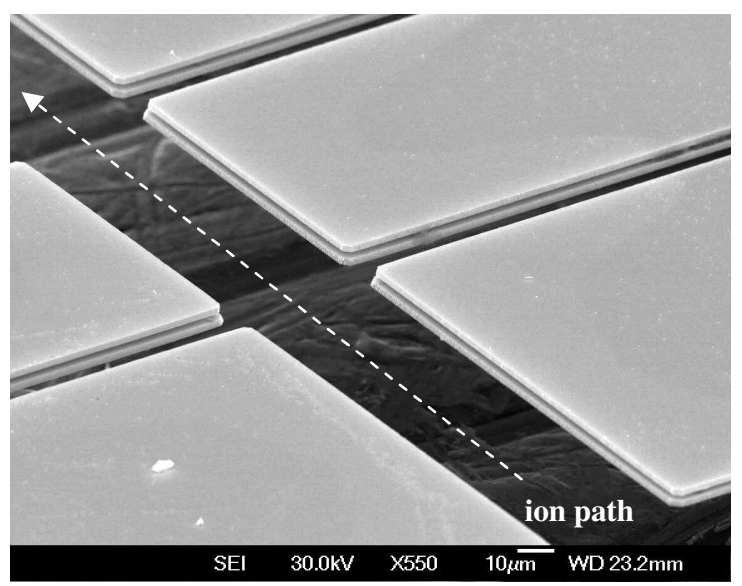

Figure 6. SEM image of quadrupole mass spectrometer. The electrodes are formed from the edge of cantilevered GaAs plates forming a quadrupole field down the instrument axis.

Vacuum-Arc Microthrusters. MTU is working with Alameda Applied Sciences, Inc. to implement vacuum arc microthrusters for 3-axis attitude control on a nanosatellite. The vehicle, HuskySat, is being developed by MTU students under the University Nanosat Initiative for launch in 2006.

Hollow-cathode Characterization. Electrostatic probe and LIF diagnostics are ongoing in an attempt to understand potential distribution and current attachment within a hollow-cathode/anode mock-up representative of an ion thruster discharge chamber. 


\section{Princeton University}

E. Y. Choueiri

Electric Propulsion and Plasma Dynamics Lab

Princeton, NJ 08543

choueiri@princeton.edu

A detailed critical history of the first 50 years of electric propulsion has been completed [1]. It reveals, among other new findings, the seminal role played by Robert $\mathrm{H}$. Goddard as early as 1906.

A new inductive thruster concept developed at EPPDyL has been under investigation. In this thruster, named the FARAD (Faraday Accelerator with Rf-Asissted Discharge), a current pulsed through a flat, spiral coil, similar to the type found in the PIT, inductively accelerates a plasma. However, unlike the PIT, ionization in the FARAD is accomplished in a separate helicon discharge and the acceleration relies on both applied and induced Lorentz forces. This decoupling of the ionization and acceleration processes allows for the scaling of FARAD down to much smaller sizes and lower energy levels than the PIT. The plasma is passively channeled to the acceleration stage using an applied magnetic field and. Recent results from a proof-of-concept experiment demonstrate current sheet production and acceleration at low energies (5-80J) (AIAA-2004-3940) [2].

Work continues towards the understanding of the fundamentals of discharge initiation in pulsed plasma thrusters. Current PPT designs, which use sparkplugs to initiate their discharges, as well as a recently proposed discharge initiation system[3], that uses laser pulses to thermionically release electrons into the electrode gap, both employ pulses of electrons to achieve a breakdown at an undervoltage. Using theoretical, numerical, and experimental tools, the questions of how large an electron pulse is required to achieve breakdown under a given set of conditions, and how the discharge initiation in PPTs can be characterized are addressed.

An investigation of the impact of plasma leakage from the current sheet on the performance of electromagnetic pulsed plasma thrusters is underway [4] (AIAA-20043463). The impulse is divided between the momentum of the sheet and a plasma wake. Recent experiments have measured the total impulse with a momentum plate and the sheet impulse with interferometry. An analytical model of the discharge propagation predicts the sheet and wake impulse and shows that as much as $70 \%$ of the impulse can be in the wake.

Researchers at EPPDyL have recently derived the sufficient and necessary conditions for a new higly efficient ion energization mechanism by beating electrostatic waves (BEW) propagating[5-8]. The
Beating Waves Experiment (BWX) was recently constructed to study this new mechanism [9]. Recently beating Electrostatic Ion Cyclotron (EIC) waves were successfully launched perpendicularly to the applied magnetic field (AIAA-2004-4095) and shown the measured dispersion relations to be in excellent agreement with theory [10]. Evidence of ion energization is being sought with a new Laser Induced Fluorescence system.

Various research activities on MPDTs continue at EPPDyL yielding new publications including: a study of cargo and piloted missions study to Mars [10], a selfconsistent hollow-cathode theory for lithium MPDTs that is the most comprehensive theory to date and includes detailed atomic physics and plasma effects and that compares favorably with experiments[11], and highly realistic[12] simulations of MPDT flows including lithium [13].

\section{References}

1. E. Y. Choueiri. A critical history of electric propulsion: The first 50 years (1906-1956). Journal of Propulsion and Power, 20(2):193--203, 2004. Also AIAA-2004-3334 at JPC 2004.

2. K.A. Polzin, and E.Y. Choueiri, "Faraday Acceleration with Radio-frequency Assisted Discharge (FARAD) Concept", 40 $0^{\text {th }}$ JPC. AIAA2004-3940.

3. J. Berkery and E.Y. Choueiri. Laser Discharge Initiation for Gas-Fed Pulsed Plasma Thrusters. In 37th Joint Propulsion Conference, Salt Lake City, UT, July 8-11, 2001. AIAA-2001-3897

4. J.W. Berkery, and E.Y. Choueiri, "Canted Current Sheet Mass Leakage and its Impact on Pulsed Plasma Thruster Performance.

5. E.Y. Choueiri and R. Spektor "Coherent ion acceleration using two electrostatic waves", in $36^{\text {th }}$ AIAA/ASME/SAE/ASEE Joint Propulsion Conference, Huntsville, Al, 2002. AIAA-20003759.

6. R. Spektor and E.Y. Choueiri "Ion Acceleration by Beating Electrostatic Waves: Criteria for Regular and Stochastic Acceleration", IEPC-01-209.

7. R. Spektor and E.Y. Choueiri "Effects of Ion Collisions on Ion Acceleration

by Beating Electrostatic Waves.", IEPC-03-65.

8. R. Spektor and E.Y. Choueiri "Ion acceleration by beating electrostatic waves: Domain of allowed acceleration", Phys.Rev.E, 69, 046402, 2004.

9. R. Spektor and E.Y. Choueiri, "An Experiment for Studying Ion

Acceleration by Beating Electrostatic Waves", in $38^{\text {th }}$ Joint Propulsion Conference, Indianapolis, IN, 2002. AIAA-2002-3801. 
10. K. Sankaran, L.D. Cassady, A.D. Kodys, and E.Y. Choueiri. A survey of propulsion options for cargo and piloted missions to mars. Annals of the New York Academy of Science, 1017:450--467, 2004.

11. L. D. Cassady, and E. Y. Choueiri. Lithium-fed Single-channel Hollow Cathode Theory. In 40th AIAA Joint Propulsion Conference, number AIAA 2004-3431, Fort Lauderdale, FL, USA, 2004. July 7-10.

12. K. Sankaran S. Jarding, and E.Y. Choueiri. Simulation of self-field MPDTs and Comparison to Experiments Accepted for publication in the Journal of Propulsion and Power, March 2004.

13. K. Sankaran, PhD thesis, September 2004.

\author{
Princeton University \\ N. J. Fisch and Y. Raitses \\ Princeton Plasma Physics Laboratory \\ Princeton, NJ 08543 \\ nfisch@pppl.gov
}

Cylindrical Hall thrusters (CHT) have lower surface-tovolume ratio and, thus, seem to be more promising for scaling down [1]. A 2.6 diameter CHT was already shown to achieve the anode thruster efficiency of 15$23 \%$ in the power range 50-200 W. Results of plasma measurements inside the thruster channel were also reported in our previous works. Electron cross-field transport and ion acceleration in this thruster was studied through the analysis of this experimental data and Monte Carlo simulations of electron and ion dynamics in the thruster channel. We found that in order to explain the observed discharge current, the electron anomalous collision frequency $v_{\mathrm{B}}$ has to be on the order of the Bohm value, $v_{\mathrm{B}} \sim \omega_{\mathrm{r}} / 16$. The electron distribution function $(\mathrm{EDF})$ obtained in the simulations is depleted at high energy due to electron loss at the walls, thus indicating that the contribution of secondary electrons to cross-field transport is likely insignificant. The plasma density peak observed at the axis of the $2.6 \mathrm{~cm}$ cylindrical Hall thruster is likely to be due to the convergent flux of ions, which are born in the annular part of the channel and accelerated towards the thruster axis [2]. The model is now applied for optimization of the $2.6 \mathrm{~cm}$ thruster.

Ongoing research is also focused on characterization of magnetic insulation properties of the Hall thruster plasma. Using a novel low disturbing segmented shielded probe [3], which is introduced to the thruster by a fast positioner, we conducted measurements of the plasma potential and electron temperature distributions along the median a $2 \mathrm{~kW}$ annular Hall thruster in the discharge voltage range of 200-700 V. In addition, we used stationary biased cylindrical probes placed the outer channel wall. Results of plasma measurements with different probe techniques were in an agreement. By plotting the electron temperature as a function of the plasma potential we found a linear dependence of the electron temperature gradient on the electric field in the acceleration region of this thruster (Figure 7). This plot illustrates a simplified electron energy equation in the form, $T_{e}=\beta \phi$. The coefficient $\beta$ is constant in the acceleration region $(\sim 0.1)$ and smaller than can be expected from ohmic heating (2/5). This indicates a yet unexplained energy loss term proportional to the electric field. Furthermore the linear relationship holds both inside and outside the thruster for electron temperature up to $40 \mathrm{eV}$, indicating that secondary electron emission do not have a significant effect on the electron temperature at the discharge voltages $<400 \mathrm{~V}$.

Low-disturbing probe measurements of the plasma properties in the near anode region of a $2 \mathrm{~kW}$ Hall thruster allowed first experimental identification of both electron-repelling (negative fall) and electron-attractive (positive fall) anode sheaths in Hall thrusters [4]. An interesting new phenomenon revealed by the probe measurements is that the anode fall changes from negative to positive with a reduction of the anode collecting area by a dielectric (oxide) coating, which appears on the anode surface during the course of normal Hall thruster operation. Interesting the amplitude of low frequency discharge oscillations tends to decrease with the coating. The results of these studies may have some practical importance for the anode, gas feed systems and facility designs especially for lifetime thruster tests.

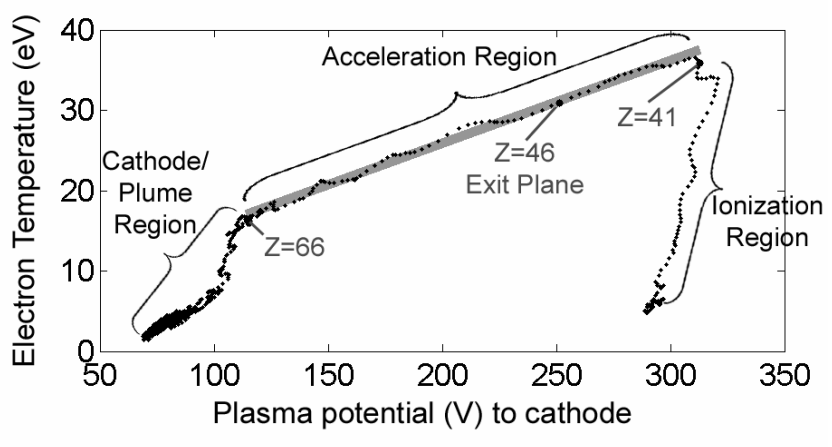

Figure 7. $\mathbf{T}_{\mathrm{e}^{-}} \phi$ plot for a $2 \mathrm{~kW}$ Hall thruster operated at $300 \mathrm{~V}$ discharge voltage, $5.0 \mathrm{mg} / \mathrm{s}$ xenon flow rate, and 4.56 $A$ discharge current. $Z$ indicates the distance from the anode [3].

\section{References}

1. Y. Raitses and N. J. Fisch, Phys. Plasmas, 8, 2579, 2001

2. A. Smirnov, Y. Raitses and N. J. Fisch, AIAApaper 2004-4103, July 2004. 
3. D. Staack, Y. Raitses and N. J. Fisch, Appl. Phys. Let., 84, 3028, 2004

4. L. Dorf, Y. Raitses and N. J. Fisch, AIAA-paper 2004-3779, July 2004.

\section{Electric Propulsion Efforts at Texas Tech John Mankowski, James Dickens, and Greg Foster Center for Pulsed Power and Power Electronics Lubbock, TX 79409 \\ j.mankowski@coe.ttu.edu}

Current efforts at the Center for Pulsed Power and Power Electronics $\left(\mathrm{P}^{3} \mathrm{E}\right)$ Texas Tech University focus around testing and evaluating the performance of lowpower Hall thrusters. To this end, a micro-thrust stand was constructed. In addition, a new data acquisition and software system was constructed for life testing of these low power thrusters.

Testing is being performed on an SPT-type, $100 \mathrm{~W}$ Hall thruster. Typical flow rate, thrust and specific impulse is $4 \mathrm{sccm}, 15 \mathrm{mN}$, and $1300 \mathrm{sec}$, respectively. An image of the thruster is shown in Figure 8.

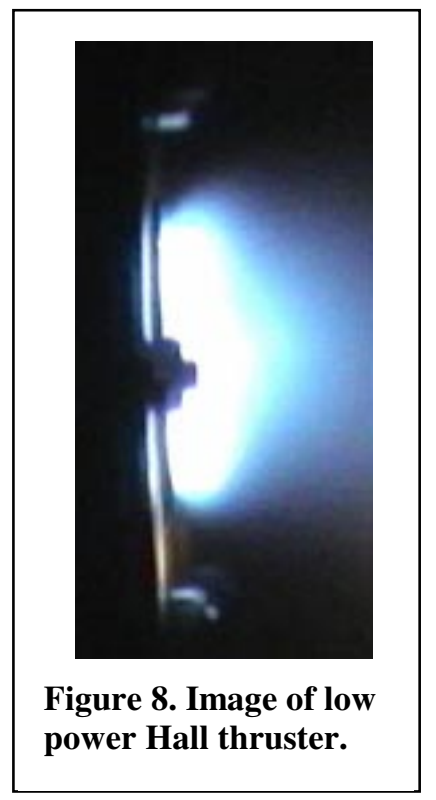

Thruster performance was evaluated in the $\mathrm{P}^{3} \mathrm{E}$ Large Space Simulation Facility. The facility is an 8 ' diameter stainless steel chamber with both diffusion and cryogenic pumping capability. The large diameter of the chamber made it possible to employ a simple, pendulum-type thrust stand and still achieve thrust measurements with a high level of accuracy, on the order of $300 \mu \mathrm{N}$.
The stand measures force created by the thruster indirectly by detecting linear displacement. This is done with six main components, shown in Figure 9. First, there is the pendulum rod, about one meter long and attached to the top of the vacuum chamber with a knife edge pendulum configuration. The second piece is the target rod, which is a piece of 0.25 " quartz tubing that slides through the pendulum rod and is secured with snap rings. This rod extends to the edge of the chamber to a viewport and provides a target for the limited range of the laser range finder. The Laser Range Finder (LRF) is a Banner LG10A65PU Laser gauging sensor that is mounted to the exterior of the viewport. This LRF boasts an accuracy of 20 microns with a $4 \mathrm{~Hz}$ acquisition rate. The thruster is mounted to the bottom of the pendulum with a custom bracket. Extending from the thruster is a calibration string that runs over a Teflon pulley and attaches to a chain. This string has calibrated weights attached, and when moved up and down, applies varying amounts of weight to the thruster, allowing for calibration. Finally, the cathode is placed at a stationary position behind the thruster.

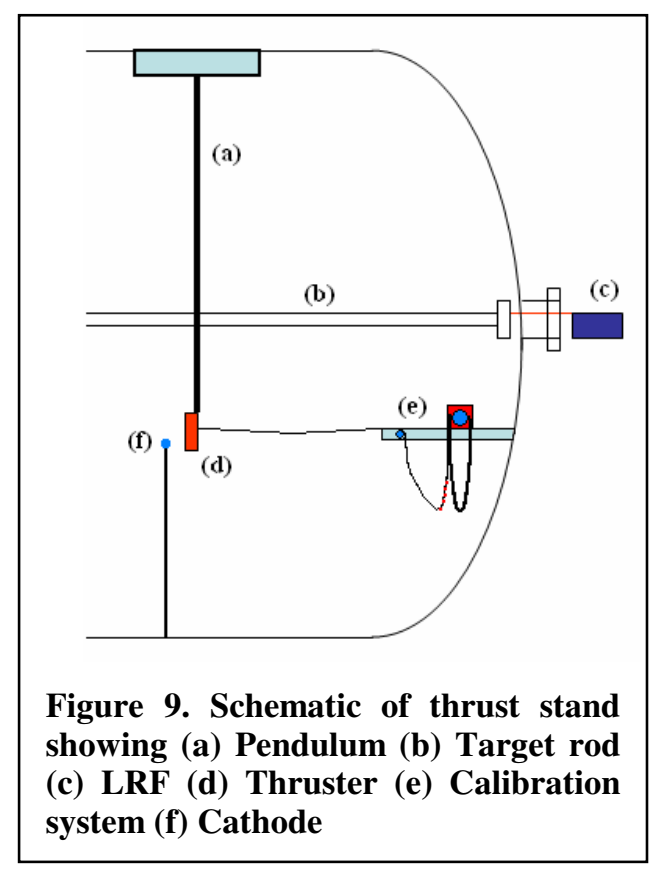

At present, over 300 hours of testing has been conducted. The current goal is for 1000 hours of lifetime testing. Future goals are to improve performance of these low-power thrusters by redesign and continued testing. 


\section{University of Illinois \\ E. Antonsen, F. Rysanek, J. Laystrom and R. Burton \\ Department of Aerospace Engineering \\ frysanek@uiuc.edu}

Surface Temperature Measurements (Antonsen)

The University of Illinois and the Air Force Research Laboratory are collaborating to apply high-speed Mercury Cadmium Telluride ( $\mathrm{HgCdTe}$ ) photovoltaic detectors to a micro-pulsed plasma thruster (PPT) to explore the surface temperature profile throughout the discharge process [1]. For an ablative arc over Teflon $^{\mathrm{TM}}$, the infrared emission is explored during and after the discharge. During the discharge, plasma emission masks the surface emission signal in the current experimental setup. However, real-time surface temperature measurements are made after the current pulse ends to examine propellant cooling and late-time ablation characteristics of the Teflon propellant. Calibration is performed on heated Teflon, experimentally accounting for temperature dependent variations in emissivity. Time dependent measurements are taken of the fuel face and used to determine a surface temperature profile after the arc. Photographs of the arc breakdown are taken with a gated, intensified camera capable of 5 ns shutter times allowing visual interpretation of the arc emission in terms of nonaxisymmetric arc spoking effects. The data are analyzed to calculate Teflon vapor pressure and expected impulse bit contribution of the neutral vapor liberated from the fuel face during the ablative cooling process. Molecular flux and mass loss are calculated after the pulse to characterize late-time ablation. The cooling profile is also compared with predictions from the Keidar-Boyd model. Model comparisons indicate a high degree of agreement with experimental measurements of the cooling profile.

\section{References}

1. Antonsen, E. L., "Propellant Surface Temperature and Plume Characteristics of Micro-Pulsed Plasma Thrusters," Ph.D. Dissertation, University of Illinois Urbana-Champaign, 2004.

\section{Macroparticle Characterization in Vacuum Arcs}

(Rysanek)

Vacuum arc thrusters (VATs) are being developed [1] to provide a highly scalable micro-propulsion system for use on small $<100 \mathrm{~kg}$ satellites. VATs operate by producing an arc discharge between two electrodes, and produce thrust by emission of neutrals and ions from the negative electrode. Ion velocities have been measured up to $15 \mathrm{~km} / \mathrm{s}$, (aluminum) corresponding to energies over $30 \mathrm{eV}$. Macroparticles in the form of molten droplets are also emitted from the cathode.
Macroparticles range in size up to tens of micron in diameter. They have a velocity less than $1000 \mathrm{~m} / \mathrm{s}$ depending on arc characteristics and cathode material [2]. Among the explanations suggested for macroparticle formation are: 1) fracture of cathode material by thermoelastic stresses [3], 2) expulsion of material due to the local electric field, and [4] 3) expulsion due to plasma pressure [5]. A model by Keidar indicates that the macroparticles emitted from the cathode are charged negatively. An experiment comparing the number of macroparticles collected on a floating potential substrate as opposed to one biased down to -1000 Volts seems to support the model [6]. The University of Illinois is designing an experiment that will measure the charge of macroparticles emitted from a vacuum arc.

\section{References}

1. J. Schein, et. al, "Low Mass Vacuum Arc Thruster System For Station Keeping," Alameda Applied Scientific Corporation, IEPC-01-228, 27th Int. Electric Propulsion Conf., Pasadena CA, Oct 2001.

2. S. Shalev, R. L. Boxman, and S. Goldsmith. Velocities and emission rates of cathode-produced molybdenum macroparticles in a vacuum arc. J. Appl. Phys., 58(7): 2503-2507, 1985.

3. V.I. Rakhovskii and A. M. Yagudaev. Electrode erosion mechanism in a pulsed vacuum arc. Sov. Phys. Tech. Phys., 14: 227-230, 1969.

4. N. N. Lebedev and I. P. Skal'skaya. Force acting on a conducting sphere in the field of a parallel plate condenser, Sov. Phys. Tech. Phys.,7: 268-270, 1962.

5. G. M. McClure. Plasma expansion as a cause of metal displacement in vacuum-arc cathode spots. J. Appl. Phys., 45: 2078-2084, 1974.

6. M. Keidar, R. Aharonov, and I.I. Beilis, Influence of an electrical field on the macroparticle size distribution in a vacuum arc, J. Vac. Sci. Technol. A, 17(5) 1999, pp. 3067-3073.

Advanced Pulsed Plasma Thruster (Laystrom)

PPT-11 (Figure 10) is an advanced side-fed coaxial design that maximizes thruster performance while minimizing the thruster mass [1,2]. Four Teflon propellant bars are fed radially through the cathode and rest against a central anode. The total thruster wet mass is $318 \mathrm{~g}$ including the useable propellant mass of $70 \mathrm{~g}$. Up to four Unison mica capacitors, totaling $82 \mu \mathrm{F}$, provide energy storage. The result of this development is a flight-capable thruster with a specific thrust of 21 $\mu \mathrm{N}-\mathrm{s} / \mathrm{J}$, a total thrust of $1.7 \mathrm{mN}$, and an $\mathrm{I}_{\mathrm{sp}}$ of $1200 \mathrm{~s}$. The highly repeatable thrust is approximately $70 \%$ electromagnetic. The total impulse bit is $1.4 \mathrm{mN}-\mathrm{s}$. The thruster operates at $85 \mathrm{~W}$ with a thrust efficiency of $12 \%$. A typical system mass is $8.27 \mathrm{~kg}$ which includes 
four thrusters, propellant, spark plugs, striplines, and 70 J capacitors. PPT-11 geometry studies have been performed. Three propellant geometries, five anode shapes, and three anode materials were tested resulting in a configuration that maximizes thrust efficiency and overall thruster performance. PPT-11 is modular such that it can be utilized in a variety of small spacecraft for various mission requirements.

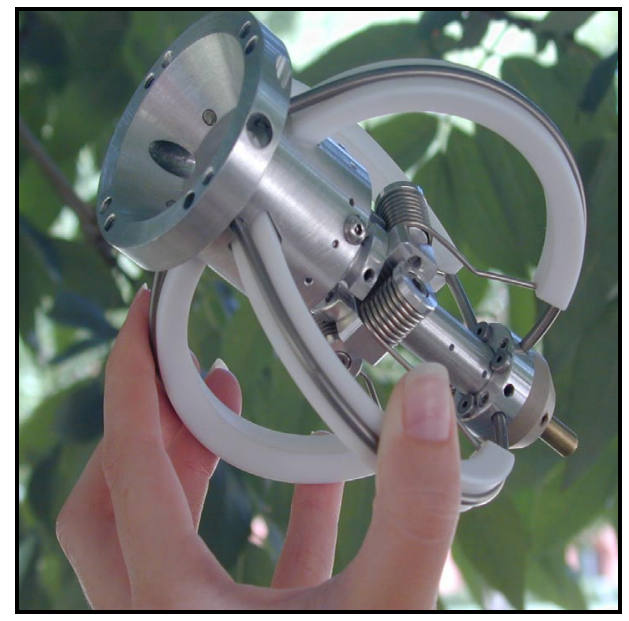

Figure 10. PPT-11

\section{References}

1. Laystrom, J. K., Burton, R. L., Benavides, G. F., "Geometric Optimization of a Coaxial Pulsed Plasma Thruster," 39th AIAA Joint Propulsion Conf., Paper AIAA-2003-5025, Huntsville, AL, July 2003.

2. Laystrom, J. K., "Propellant and Central Electrode Optimization of a Coaxial Pulsed Plasma Thruster," M.S. Thesis, University of Illinois at UrbanaChampaign Graduate College, June 2004.

\section{The University of Michigan Iain D. Boyd \\ Department of Aerospace Engineering Ann Arbor, MI 48109-2140 \\ http://hpcc.engin.umich.edu/CFD/research/NGPD/}

Our research involves the development of advanced computer models for analysis of plasma and gas flows in electric propulsion (EP) systems. We are developing models of EP devices to aid in thruster design and to assess lifetime issues. We are developing models of the plumes from EP devices partly to validate the device models, and also for assessment of spacecraft integration issues. Our research is sponsored by AFOSR, NASAGRC, NASA-JPL, the Air Force Research Laboratory, the Boeing Company, and DOE. A brief summary of our current work is given below.

\section{Hall Thrusters}

a) A detailed fluid electron model was developed and applied to compute the plume of the BHT-200 [1]. The model solves continuity, momentum, and energy equations for the electrons coupled with a particle description of the heavy species. Excellent agreement is obtained with probe and LIF measurements of the thruster plume.

b) Development continues of a detailed 2d, unsteady model of the acceleration channel and near-field plume for a Hall thruster [2]. Work is in progress to analyze the NASA-173M1 Hall thruster with both xenon and krypton propellant.

c) Presheath structures and near-wall effects in the acceleration channel of an anode layer thruster were modeled using a hydrodynamic approach [3].

d) Simplified 1d Hall thruster erosion models were developed and compared with measured data [4].

Ablative Plasma Thrusters

a) Work continues in the modeling of plumes from micro-laser-ablation thrusters. A new model for transmission-mode operation was developed and the resulting plume simulation results compared very well with experimental data [5].

b) Development of detailed models of micro-pulsed plasma thrusters continues. Analysis was performed to determine the optimal energy level for a given micro-PPT [6]. When the discharge energy is small, propellant charring limits the operational life of the thruster. High energy leads to discharge constriction on the positive electrode and causes azimuthal nonuniformity. Simulated results are compared with experimental data from AFRL and good agreement was found [6].

Ion Thrusters

1. A PIC-DSMC grid optics code continues to undergo development and is being applied to analyze NASA's NEXT ion thruster [7]. In addition, a mesh-free treecode algorithm is being developed [8] that may provide superior geometric accuracy than traditional meshed PIC codes allow.

2. A detailed PIC-DSMC model was developed and applied to describe the plume from the T6 hollow cathode and compared with experimental measurements [9].

\section{Additional Projects}

a) Development continues of a code for simulating operation of electric propulsion thrusters in a vacuum chamber. The sticking coefficient of xenon on gaseous helium cryo-panels was determined through direct comparisons of simulation results 
with the residual pressure measured in PEPL at the University of Michigan [10].

b) Models are being developed to predict the dynamics of droplet formation and ion extraction from the needle of a FEEP thruster.

c) Analysis is being performed of plasma interaction with probes employed in the plumes of electric propulsion devices. The primary goals are to aid in the design and development of such probes.

\section{References}

1. Boyd, I.D. and Yim, J.T., "Modeling of the NearField Plume of a Hall Thruster," Journal of Applied Physics, Vol. 95, 2004, pp. 4575-4584. (see also AIAA-2004-3952)

2. Koo, J.W., Keidar, M. and Boyd, I.D., "Boundary Conditions for a 2D Stationary Plasma Thruster Model," AIAA Paper 2004-3781, July 2004.

3. Keidar, M., Boyd, I.D. and Beilis, I.I., "Modeling of a High-Power Thruster with Anode Layer," Physics of Plasmas, Vol. 11, 2004, pp. 1715-1722.

4. Manzella, D.H., Yim, J.T., and Boyd, I.D., "Predicting Hall Thruster Operational Lifetime," AIAA Paper 2004-3953, July 2004.

5. Keidar, M., Boyd, I.D. Luke and Phipps, C., "Plasma Generation and Plume Expansion for a Transmission-Mode Micro-Laser Plasma Thruster," Journal of Applied Physics, Vol. 95, 2004, accepted.

6. Keidar, M. and Boyd, I.D., "Optimization Criteria for a Micro Pulsed Plasma Thruster," AIAA Paper 2004-4701, July 2004.

7. Emhoff, J.W. and Boyd, I.D., "Progress in Next Ion Optics Modeling," AIAA Paper 2004-3786, July 2004.

8. Christlieb, A., Emhoff, J.W. and Boyd, I.D., "Plasma Simulations for Arbitrary Domains with Applications to Ion Optics," $24^{\text {th }}$ International Symposium on Rarefied Gas Dynamics, Bari, Italy, July 2004.

9. Boyd, I.D. and Crofton, M.W., "Modeling the Plasma Plume of a Hollow Cathode," Journal of Applied Physics, Vol. 95, 2004, pp. 3285-3296.

10. Walker, M.L.R., Gallimore, A.D., Boyd, I.D., and Cai, C., "Vacuum Chamber Pressure Maps of a Hall Thruster Cold Flow Expansion," Journal of Propulsion and Power, Vol. 20, July-August, 2004.

\author{
The University of Michigan \\ Alec Gallimore \\ Department of Aerospace Engineering \\ Ann Arbor, MI 48109-2140 \\ alec.gallimore@umich.edu
}

Over the past year, the Plasmadynamics and Electric Propulsion Laboratory (PEPL) has conducted a wide array of electric propulsion research activities that were sponsored by the AFOSR, AFRL, Jet Propulsion Laboratory, and NASA Glenn Research Center. Graduate student and post-doctoral support was provided by grants from the abovementioned organizations as well as from the Department of Defense, the François-Xavier Bagnoud Foundation, the Michigan Space Grant Consortium (NASA), and the National Science Foundation. A summary of our work over the past year follows.

- We used our high-speed reciprocating probe system (HARP) to measure Te, Ne, EEDF, and plasma potential in the discharge chamber of an NSTARderivative, ring-cusp xenon ion engine (AIAA2004-3794). Through the use of HARP, we have been able to provide unprecedented insight on the structure of the plasma near the discharge cathode of an ion engine during beam extraction.

- We have developed a new Faraday probe filtering scheme that employs a transverse magnetic field to deflect low-energy, charge-exchange ions from the collector (AIAA-2004-3948). We have made a series of comparisons between nude, gridded, and magnetically-filtered Faraday probes.

- We are investigating the influence of facility effects on Hall cluster operation. Our paper AIAA-20043767 shows how facility effects influence the measured performance of twin P5 Hall thrusters over a range of chamber pumping speeds and cluster power levels.

- We have completed a critical phase of our investigation on high-specific impulse, single-stage Hall thrusters with the NASA-173M series. Our paper AIAA-2004-3602 provides an analysis on the factors that contribute to thruster performance. We identify the suppression of excessive electron backstreaming through the utilization of a magnetic lens topology as the principal reason the 173M series thrusters achieve high efficiency at specific impulses of and above $3000 \mathrm{~s}$.

- We developed and characterized the NASA-173GT gridless ion thruster, which is a hybrid between an ion engine and a Hall thruster. While the performance of the $173 \mathrm{GT}$ is less than that of the $173 \mathrm{M}$, it does demonstrate the concept of using a somewhat independent ionization mechanism with a Hall accelerator stage. Our findings will be 
presented at this conference in paper AIAA-20043936.

- We have developed a multi-cathode discharge chamber in support of NASA-GRC's HiPEP ion thruster development program. The purpose of this work is to utilize the sequential operation of hollow cathodes to achieve long thruster life. We conducted an extensive magnetic field design procedure in developing our version of the rectangular discharge chamber. The discharge chamber should be functional by the end of the summer. Our experiments will focus on measuring ion production cost and uniformity, discharge chamber plasma properties (with HARP), and assessing the erosion of the dormant cathodes.

- We performed a series of NASA-173Mv1 performance measurements with krypton propellant. Anode efficiency peaked at $60 \%$ (at $2900 \mathrm{~s}$ and 8 $\mathrm{kW}$ ) while anode specific impulse reached $4300 \mathrm{~s}$ at an anode efficiency of just over $58 \%$ at $6 \mathrm{~kW}$. We will begin HARP discharge channel measurements on the v1 for both xenon and krypton propellants this summer.

Research Highlight: The NASA-173GT hybrid thruster development program-a work in progress The NASA Glenn Research Center and the University of Michigan's Plasmadynamics and Electric Propulsion Laboratory have built and tested a hybrid Hall/ion thruster. The design and characterization results of this laboratory-model gridless ion thruster with closed electron drift acceleration (NASA-173GT) are presented in AIAA-2004-3936. The impetus behind this work is to gain an improved understanding of the processes within a high-voltage Hall thruster beyond the single-, twostage, or segmented-anode work previously conducted. The 173GT incorporates the ionization source of an ion engine-a DC discharge with a hollow cathode in a ring-cusp magnetic field topology in this case-and the acceleration zone of a closed-drift Hall thruster (lens magnetic field topology).

The 173GT hybrid Hall/Ion thruster was successfully operated in single- and two-stage modes. The ion production cost of the ionization stage is as low as 210 $\mathrm{eV} /$ ion. Performance and plume measurements indicate that the 173GT operates with large electron backstreaming in both single and two-stage configurations. This large electron leakage current limits the acceleration efficiency of the thruster to a maximum of $36 \%$ for the two-stage configurations. Physical observation of the thruster under operation along with gridded Faraday probe measurements suggest that the electron Hall current may intersect the four "flux shunts" that are used to provide the magnetic lens topology in the acceleration stage. Several possible improvements to the thruster design, such as the removal of the flux shunt or lengthening of the Hall current zone, would most likely improve the NASA-173GT performance considerably.

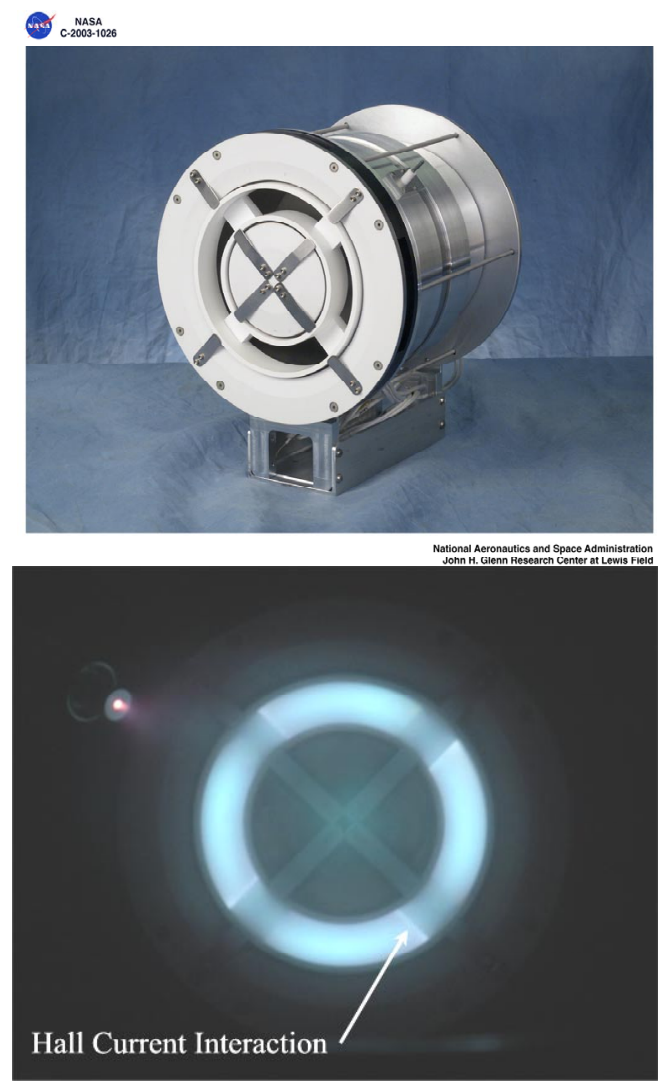

Figure 11: Studio image and picture of the NASA173GT in two-stage operation. Note evidence for Hall current interaction with the magnetic circuit flux shunts, which are encased in boron nitride.

\author{
Virginia Tech \\ Joseph J. Wang \\ Dept. Aerospace and Ocean Engineering \\ Virginia Polytechnic Institute and State University \\ Blacksburg, VA 24061-0203 \\ jowang@aoe.vt.edu
}

Electric propulsion research activities at Virginia Tech in the past year include continued experimental investigation of low energy ion sputtering in ion thrusters, continued development of electric propulsion modeling tools, and simulation studies performed in support of the DAWN mission and future solar sail missions $[1,2,3,4,5,6]$.

A highlight of our research is the development of the DRACO code, supported by Air Force Research Lab at Edwards AFB under the COLISEUM project. DRACO 
is a multi-purpose particle simulation software for modeling electric propulsion and spacecraft-plasma interactions. DRACO contains three simulation modules to provide different levels of sophistication in modeling (Figure 12). The QN-PIC module, where the plasma is assumed to be quasi-neutral and the sheath is ignored, is intended for quick calculations (within minutes of computing time). The DADI-PIC module, a standard particle-in-cell (PIC) code using a finite-difference formulation to solve the electric field, is designed for problems with relatively simple geometric conditions. The IFE-PIC module, based on a new algorithm using an immersed finite element formulation[1], is designed to perform high-fidelity simulations of problems involving complex geometric and material boundary conditions while at the same time retaining the computational speed of a standard PIC code. As the purpose of DRACO is to solve real engineering problems, DRACO reads in spacecraft configurations defined by commercial CAD tools. In addition to standard graphics visualization, a new cross-platform visualization package, capVTE, is also developed to provide immersed visualization for users with access to virtual reality systems and/or collaborative visualization for remotely connected users to interact with each other over the same data objects. Hence, as shown in Figure 12, the combined DRACO, capVTE, and CAD package provides a prototype virtual testing laboratory for electric propulsion research.

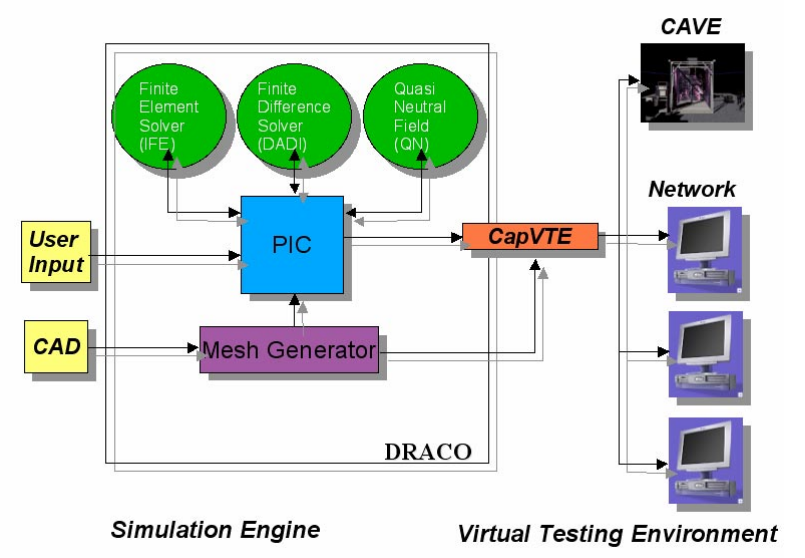

Figure 12: DRACO/capVTE: Virtual Testing Lab block diagram.

The DRACO package has been applied in a variety of electric propulsion simulation studies. Two examples using the IFE-PIC module are shown in Figures 13 and 14, respectively. Figure 13 shows an ion optics simulation. Figure 14 shows an ion thruster plume simulation for the DAWN spacecraft. The IFE-PIC utilized two different numerical meshes: PIC uses a Cartesian mesh for efficient particle push; IFE uses a non-uniform rectangular mesh embedded with tetrahedrons to resolve the details in electric field (Figure 15). We find that the use of such a numerical mesh and the immersed finite element formulation allows us not only to solve very accurately the electric field in the presence of complex boundary conditions but also achieve a computational speed-up over standard PIC codes.

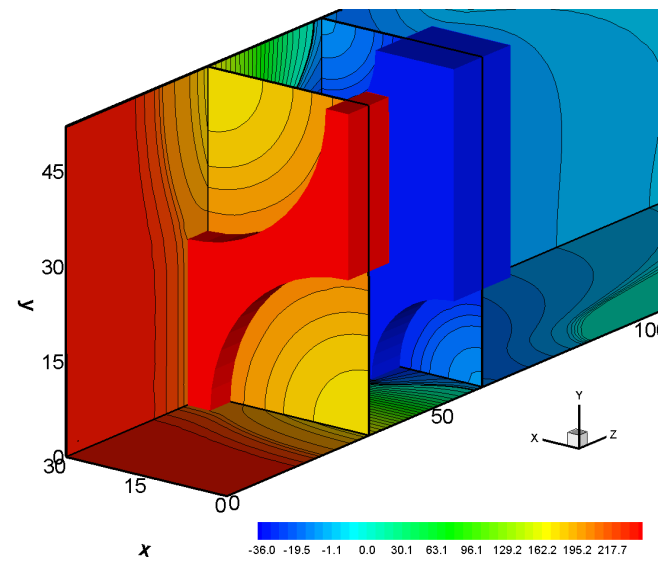

Figure 13: IFE-PIC simulations of ion optics that display electric potential contours.

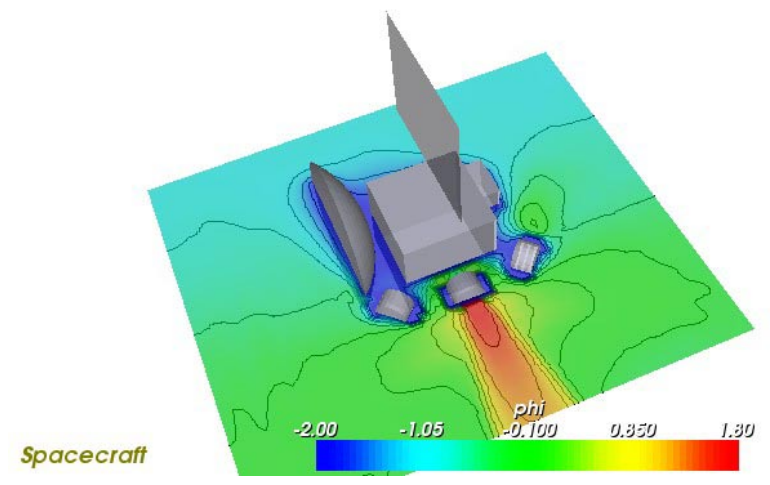

Figure 14: Ion thruster plume simulation using DRACO, showing electric potential contours. 


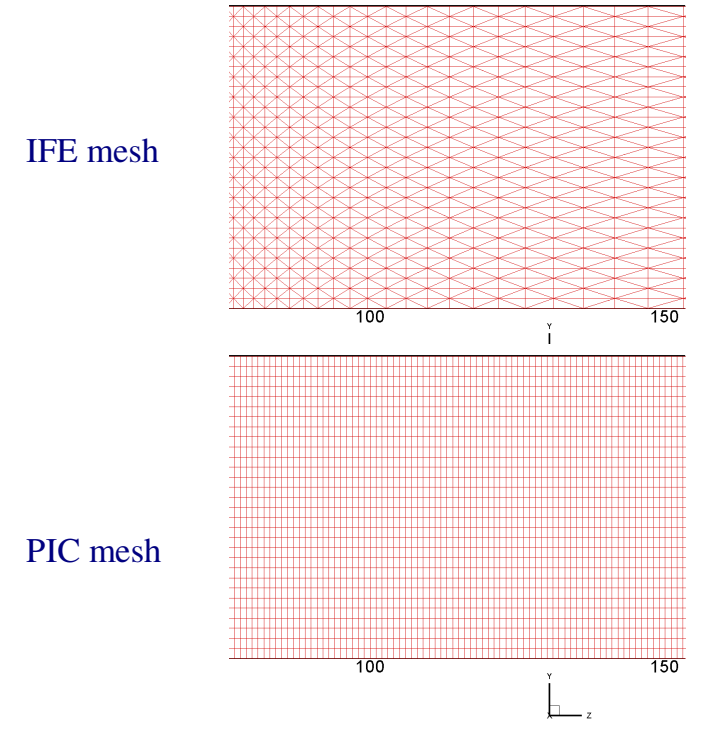

Figure 15: The non-uniform rectangular-tetrahedral IFE mesh and the Cartesian PIC mesh

\section{References}

1. R. Kafafy, T. Lin, Y. Lin, and J. Wang, "3-D Immersed Finite Element Method for Electric Field Simulation in Composite Materials", submitted to Int. Journal for Numerical Methods in Engineering, 2004.

2. L. Brieda, R. Kafafy, J. Pierru, J. Wang, "Development of the DRACO Code for Modeling Electric Propulsion Plume Interactions", AIAA 2004-3633

3. J. Wang, R. Kafafy, L., Brieda, "Modeling Multiple Ion Thruster Plume Interactions", AIAA 20043634.

4. R. Kafafy, J. Wang, "IFE-PIC Simulations of Ion Optics", AIAA 2004-3783

5. M. Nakles, J. Wang, "Further Investigations of Low Energy Ion Sputtering in Ion Thrusters", AIAA 2004-3789

6. H. Garrett, J. Wang, "Simulations of Solar Wind Plasma Flow around a Solar Sail", Proceedings of the 8th Spacecraft Charging Technology Conference, 2004.

\section{Worcester Polytechnic Institute John J. Blandino \\ Department of Mechanical Engineering Worcester, MA 01609-2280 blandino@wpi.edu}

In Prof. Blandino's group at WPI, research has continued this year in the development of diagnostics to characterize colloid thruster plumes. This experimental activity is a collaborative effort with the Jet Propulsion
Laboratory and Busek Co., which is developing the propulsion system for the NASA ST-7 Mission. Beyond demonstration on ST-7, the colloid thruster is also a candidate for the Laser Interferometer Space Antenna (LISA) Mission. The focus of this past year's effort has been the development of a charge detection mass spectrometer (CDMS). This diagnostic incorporates the capability to determine droplet charge and charge-to mass ratio independently in one compact device. These two measurements allow the determination of droplet size (for known fluid density) and the identification of different droplet populations at specific points in the plume [1].

The CDMS consist of three integrated components. The first is a charge detection tube which is used to measure droplet time-of flight and determine its charge. A passing charged droplet induces an image charge on the tube which is connected to a charge-sensitive preamplifier and shaping amplifier. The output from this detector is two voltage pulses, corresponding to the entry and exit of the droplet from the tube. The height of the measured pulse from the shaping amplifier is used to determine the droplet charge. The sensitivity (pulse height for a given charge) is calibrated with a separate circuit in which a known charge pulse is delivered to the tube. The time between the pulses corresponds to the time-of-flight of the droplet through the tube of known length.

The second component consists of a series of three screens mounted at the entrance to the charge detection tube. The center screen can be biased to repel droplets and limit the energy of droplets entering the detector. The two screens on either side of the retarding screen are grounded along with the outside of the detector. The third component is a charge accumulation cup located at the exit of the charge detection tube. The charge accumulation cup is used in conjunction with the retarding grid to determine the accelerating potential of different droplet populations.

Part of the challenge in developing this diagnostic is optimizing the tradeoff between minimizing size and maximizing sensitivity and resolution. The CDMS is cylindrical, approximately $8.1 \mathrm{~cm}$ long and $2.9 \mathrm{~cm}$ in diameter at its widest point. It is designed to be easily mounted on a probe positioning system so that droplet characteristics at different locations within the colloid plume can be characterized.

Our group has also continued to investigate mission applications of micronewton thrusters (colloid or FEEP) for formation control in earth orbit. The Gravity Recovery and Climate Experiment (GRACE) mission consists of two spacecraft launched in 2002 designed to map the earth's mass distribution with high sensitivity. 
The spacecraft are in a $485 \mathrm{~km}$, near polar, circular orbit with a nominal along track separation of $220 \mathrm{~km}$. Due to variations on the drag force on the satellites, station keeping maneuvers (using cold gas thrusters) are required every 30 to 60 days to maintain optimal separation over the five year mission. A possible follow-on mission, called EX-5, has been under consideration and could use thrusters to provide a "dragfree" platform for sensors by continuously correcting for accelerations due to drag. We have investigated the feasibility of micronewton thrusters to fulfill this role through simulations which propagate the orbits of the two spacecraft subject to perturbing accelerations due to drag. A realistic atmospheric density model is used in order to capture differential acceleration to the different spacecraft orbits. A simple closed loop control system is implemented which uses feedback of Cartesian position and velocity state errors [2].

\section{References}

1. Sartorelli, R., Blandino, J., and Gamero-Castaño, M., "Development of a Charge Detection Mass Spectrometer for Study of Colloid Thruster Plumes," AIAA-2004-4849, 40 ${ }^{\text {th }}$ AIAA Joint Propulsion Conference, July 2004.

2. St. Rock, B., Blandino, J., and Demetriou, M., "Application of Micronewton Thrusters for Control of Multispacecraft Formations in Earth Orbit Paper Number," AIAA-2004-3811, 40 ${ }^{\text {th }}$ AIAA Joint Propulsion Conference, July 2004.

\section{Worcester Polytechnic Institute Nikolaos A. Gatsonis \\ Department of Mechanical Engineering Worcester, MA 01609-2280 gatsonis@wpi.edu}

Research activities in Prof. Gatsonis' group encompass development of advanced plasma diagnostics, plume modeling, and micropropulsion modeling. Research was sponsored by the AFOSR, NASA Glenn Research Center and the Massachusetts Space Grant Consortium.

Modeling and Control of Pulsed Plasma Thrusters While investigations of the plasma dynamics in the electrode region as well in the plume region of a PPT have been numerous the prospects of flow control aiming in performance optimization have not been addressed so far. In AIAA- 2004-3464 we explore the possibilities of flow-control of an ablative parallel-plate PPT. A slug-model for the plasma flow is used to develop a feedback control strategy that allows the maximation of the objective exhaust velocity.
Modeling of Ion Beam Neutralization

A fundamental area in plasma physics and electric propulsion is the coupling of the electrons to an ion beam, such as from an electric propulsion device. Under investigation are the coupling mechanisms between the beam, neutralizer, and background plasma. Initial simulations of an ion beam in background plasma with neutralization are investigated with a 3D unstructured DSMC/PIC code in AIAA-2004-4121.

\section{Pulsed Plasma Thruster Diagnostics and}

\section{Characterization}

This collaborative with NASA Glenn Research Center effort entailed the novel implementation of triple and quadruple Langmuir probe diagnostics (Gatsonis et al, $2004 \mathrm{a}$; 2004b) The probe operation is referred to as current-mode and was proven suitable for the unsteady and fluctuating PPT plume. The current-mode involves biasing the probes and measuring the resulting currents without the need of obtaining a floating-voltage measurement. The current-mode probe theory incorporates advanced current collection models as well as formal error analysis.

Modeling of Plasma for Micropropulsion Applications

The research addresses fundamental mathematical and computational issues in plasma micro-flows. The research aims in the modeling of electric microthrusters (e.g. ion, hall, FEEP, resistojet) and associated devices (e.g. hollow cathodes, field emission arrays) in order to optimize basic performance characteristics. In addition the research aims in the modeling of electric microthruster plumes in order to investigate potential plume/microspacecraft interactions. The code under development uses concepts of DSMC, PIC on unstructured grids with adaptation. As an application we consider the operation of field emission (FE) arrays that can be part of electric micropropulsion devices. In Spirkin and Gatsonis (2004) we addressed issues of numerical heating in PIC computations on unstructured 3-D grids. Various alternatives of particle and force weighting schemes are considered. The problem of the collisionless suddenly expansion of plasma into vacuum is investigated. Results are compared with analytical and previous computational works.

\section{References}

1. Gatsonis N.A. and Demetriou, M.A., "Prospects of Plasma Flow Modeling and Control for a Pulsed Plasma Thruster," AIAA Paper no 2004-3464, July 2004.

2. Wheelock, A., Gatsonis, N.A., and Cooke, D.L. "Computational Modeling of Ion Beam- Neutralizer Interactions in Two and Three Dimensions, " AIAA Paper No. 2004-4121, July 2004. 
3. Gatsonis, N.A, Byrne. L., Zwahlen, Pencil, E., Kamhawi, H., "Current-Mode Triple and Quadruple Langmuir Probe Methods with Applications to Flowing Pulsed Plasmas," IEEE Transactions on Plasma Science, to be published August 2004a.

4. Gatsonis, N.A, Zwahlen, Wheelock, A., Pencil, E., Kamhawi, H., "Pulsed Plasma Thruster Plume Characterization Using a Current-Mode Quadruple Langmuir Probe Method", Journal of Propulsion and Power, Vol. 20, No. 2, pp. 243-254, MarchApril, 2004b.

5. Spirkin A. and Gatsonis, N.A., "Unstructured 3D PIC Simulations of the Flow in a Micro-Retarding Potential Analyzer," Computer Physics Communications, accepted for publication November 2003. 